\title{
Exact Solutions for nonlinear partial differential equation by modified F-expansion method
}

\author{
Najla.A.AL-Khairullah \\ Mazin.H.suhhiem \\ Department of Statistics, College of Administration and Economies \\ Sumer University \\ nalkhairalla5@gmail.com \\ Mazin.suhhiem@yahoo.com
}

Recived : 17\7\2018

Revised : 2617\2018

Accepted : $16 \backslash 8 \backslash 2018$

Available online : $25 / 9 / 2018$

DOI: $10.29304 / j q c m .2018 .10 .3 .433$

\begin{abstract}
In this Paper, we use modified F-expansion method to construct new exact traveling wave solutions of nonlinear partial differential equation, The $(2+1)$ - dimensional zoomeron equation, The obtained solutions include soliton - like solutions, trigonometric function solutions, rational solutions and exponential solutions.
\end{abstract}

Mathematics subject classification: 35-XX . 
Najla.A/ Mazin.H

\section{1- Introduction}

Nonlinear partial differential equations are used to describe many phenomena in physics and other domains. The investigation of the exact solutions of Nonlinear PDE plays an important role in the study of physical phenomena.

In applied mathematics, it has importance to obtain and search the exact solutions of these equations. Therefore, recently, a lot of efficient and accurate methods to find exact solutions for Nonlinear PDES submit by many authors includes the $\left(G^{\prime} / G\right)$ - expansion method [3], Homogeneous Balance Method [6], Darboux transformation method [8], the inverse Scattering method [1], the F-expansion method [9], the sinecosine method [7], Jacobi elliptic function [5], homotopy perturbation method [2] and several powerful methods which have been employed to obtain exact solutions.

\section{2 - Description of the Method}

Consider a general nonlinear partial differential equation, with two variables $\mathrm{x}, \mathrm{t}$

$$
P\left(u, u_{t}, u_{x x}, u_{x t}, u_{t t}, \ldots\right)=0 \quad \ldots(2-1)
$$

Where $u=u(x, t)$ is the solution of (2-1)

Step I: The transformations which are used are as follows:

$u\left(x_{1}, x_{2}, \ldots, x_{l}, t\right)=u(\delta)$

$\delta=k_{1}\left(x_{1}+k_{2} x_{2}+\cdots+k_{l} x_{l}-\lambda t\right) \ldots(2-2)$

Where $k_{1}, k_{2}, \ldots, k_{l}, \lambda$ are constants to be determined Inserting (2-2) into (2-1) change the NPDE to ODE for $u(\delta)$

$p(u, u, u ́ u, \ldots)=0$

Step II: According to the modified F-expansion method,

$u(\delta)=a_{0}+\sum_{i=1}^{N} a_{i} F^{i}(\delta)+\sum_{i=1}^{N} b_{i} F^{-i}(\delta)$

$$
\text { ... }(2-4)
$$

Where $a_{0}, a_{i}, b_{i}$ constants to be determined are $F(\delta)$ satisfies the following eq. :

$F(\delta)=A+B F(\delta)+C F^{2}(\delta) \quad \ldots(2-5)$

Where $\mathrm{A}, \mathrm{B}$ and $\mathrm{C}$ are constants to be determined.

Integer $\mathrm{N}$ can determined by considering the homogeneous balance between the governing nonlinear term(s) and highest order derivatives of $V(\delta)$ in (2-3). Given different values of A , B and $\mathrm{C}$, the different Riccati function solution , $F(\delta)$ can be obtained from (2-5) (see Table 1 ).

Step III: Substitute (2-4) into (2-3) and using (2$5)$, and collect coefficients of $F^{i}(\delta)(i=$ $-N, \ldots, N)$, then set each coefficient to zero.

Equating each coefficient of $F^{i}(\delta)$ to zero yields a system of algebraic equations for $a_{i}(i=$ $N, \ldots, 1,0), b_{i}(i=1, \ldots, N), k_{i}(i=$ $1, \ldots, m)$ and $\lambda$.
Step IV: Solve the system of algebraic equation, $a_{i}, b_{i}, k_{i}, \lambda$ can be expressed by $\mathrm{A}, \mathrm{B}$, and $\mathrm{C}$ (or the coefficients of ODE (2-3)). Substituting these results into (2-4), we can obtain the general form of travelling wave solutions to Eq. (2-3).

StepV: From Table 1, and the general form of travelling wave Solutions, we can obtained a series of Soliton - like solutions, trigonometric function Solutions and rational solutions of Eq.(2-1).

Table1. Relations between values of A, B, C and

\begin{tabular}{|c|c|c|c|}
\hline \multicolumn{4}{|c|}{$F(\delta)=A+B F(\delta)+C F^{2}(\delta)$} \\
\hline $\mathrm{A}$ & $\mathrm{B}$ & $\mathrm{C}$ & $F(\delta)$ \\
\hline 0 & 1 & -1 & $\frac{1}{2}+\frac{1}{2} \tanh \left(\frac{1}{2} \delta\right)$ \\
\hline 0 & -1 & 1 & $\frac{1}{2}-\frac{1}{2} \operatorname{coth}\left(\frac{1}{2} \delta\right)$ \\
\hline \multirow{2}{*}{$\frac{1}{2}$} & \multirow[t]{2}{*}{0} & \multirow{2}{*}{$\frac{-1}{2}$} & $\operatorname{coth}(\delta) \pm \operatorname{Csch}(\delta)$ \\
\hline & & & $\tanh (\delta) \pm i \operatorname{sech}(\delta)$ \\
\hline 1 & 0 & -1 & $\tanh (\delta), \operatorname{coth}(\delta)$ \\
\hline \multirow{2}{*}{$\frac{1}{2}$} & \multirow[t]{2}{*}{0} & \multirow{2}{*}{$\frac{1}{2}$} & $\sec (\delta)+\tan (\delta)$ \\
\hline & & & $\operatorname{Csc}(\delta)-\cot (\delta)$ \\
\hline \multirow{2}{*}{$\frac{-1}{2}$} & \multirow[t]{2}{*}{0} & \multirow{2}{*}{$\frac{-1}{2}$} & $\sec (\delta)-\tan (\delta)$ \\
\hline & & & $\operatorname{Csc}(\delta)+\cot (\delta)$ \\
\hline $\begin{array}{l}1(- \\
1)\end{array}$ & 0 & $1(-1)$ & $\tanh \delta(\cot \delta)$ \\
\hline 0 & 0 & $\neq 0$ & $\frac{-1}{c \delta+\eta}(\eta$ is anarbitrary \\
\hline $\begin{array}{l}\text { A is } \\
\text { arbit- } \\
\text { rary } \\
\text { cons- } \\
\text { tant }\end{array}$ & 0 & 0 & $\mathrm{~A} \delta$ \\
\hline $\begin{array}{l}\text { A is } \\
\text { arbit- } \\
\text { rary } \\
\text { const } \\
\text { ant }\end{array}$ & $\neq 0$ & 0 & $\frac{\exp (B \delta)-A}{B}$ \\
\hline
\end{tabular}
corresponding $F(\delta)$ in Riccati equation

3-Exact solutions to zoomeron equation

In this section we apply this method to Construct exact solution of the $(2+1)$ - dimensional zoomeron equation (see [4])

$\left(\frac{u_{x y}}{u}\right)_{t t}-\left(\frac{u_{x y}}{u}\right)_{x x}+\left(2 u^{2}\right)_{x t}=0 \quad \ldots(3-1)$ 
Where function $\mathrm{u}(\mathrm{x}, \mathrm{y}, \mathrm{t})$ is the amplitude of the relevant wave mode. This highly nonlinear equation play an important role in describing the evolution of a single Scalar field and is a convenient model to display the noval phenomena associated with boomers and trapons. If $y=t$, the $(2+1)$ - dimensional zoomeron equation reduces to its $(1+1)$ - dimensional form which can be regarded as a sub case of the boomeron equation.

By using the transformation:

$u(x, y, t)=u(\delta)$ and

$\delta=x+y-\lambda t$

Where $\lambda$ is arbitrary constant.

Substituting Eq. (3-2) with Eq. (3-1), the change will be:

$\lambda^{2}\left(\frac{\dot{u}}{u}\right)-\left(\frac{\dot{u}}{u}\right)=2 \lambda\left(u^{2}\right)=0 \quad \ldots(3-3)$

Now integrating (3-3) with respect $\lambda$ twice, we get:

$\left(\lambda^{2}-1\right) \dot{u}-2 \lambda u^{3}+k u=0 \quad \ldots(3-4)$

Where $\mathrm{k}$ is an integral constant.

Considering the homogeneous balance between $u_{\delta \delta}$ and $u^{3}$ in (3-3),

The Solution to ordinary Eq. (3-3) can be expressed be

$U(\delta)=a_{0}+a_{1} F(\delta)+b_{1} F^{-1}(\delta) \quad \ldots(3-5)$

Where $a_{0}, a_{1}$ and $b_{1}$ are constants to be determined. By Substituting (3-5) with (3-3) and using (2-5) the left - hand side of Eq.(3-5) can be converted into a finite series in $F^{j}(\delta) \quad(j=$ $-3, \ldots, 3)$.

Equating each coefficient of $F^{j}(\delta)$ to zero yields a system of algebraic equations for $a_{0}, a_{1}, b_{1}$ and $k$.

$$
\begin{gathered}
F^{-3}:\left(\lambda^{2}-1\right)\left(2 b_{1} A^{2}\right)-2 \lambda b_{1}^{3}=0 \\
F^{-2}:\left(\lambda^{2}-1\right)\left(3 b_{1} A B\right)-2 \lambda\left(3 a_{0} b_{1}^{2}\right)=0 \\
F^{-1}:\left(\lambda^{2}-1\right)\left(2 b_{1} A C+b_{1} B^{2}\right)-2 \lambda\left(3 a_{0}{ }^{2} b_{1}+\right. \\
\left.3 a_{1} b_{1}^{2}\right)+k b_{1}=0 \\
F^{0}:\left(\lambda^{2}-1\right)\left[a_{1} A B+b_{1} B C\right]-2 \lambda\left(a_{0}^{3}\right)+k a_{0} \\
\quad+6 a_{0} a_{1} b_{1}=0 \\
F:\left(\lambda^{2}-1\right)\left[a_{1} B^{2}+2 a_{1} A C\right]-2 \lambda\left[3 a_{0}^{3} a_{1}\right. \\
\left.+3 a_{1}^{2} b_{1}\right]+k a_{1}=0 \\
F^{2}:\left(\lambda^{2}-1\right)\left(3 a_{1} B C\right)-2 \lambda\left(3 a_{0} a_{1}{ }^{2}\right)=0 \\
F^{3}:\left(\lambda^{2}-1\right)\left(2 a_{1} C^{2}\right)-2 \lambda\left(a_{1}{ }^{3}\right)=0 \\
\ldots(3-6)
\end{gathered}
$$

Solving the algebraic equations (3-6), we have the following solutions of $a_{0}, a_{1}, b_{1}$ and $k$

Case I: $a_{0}=0 \quad, a_{1}=c \sqrt{\lambda-\frac{1}{\lambda}} \quad, b_{1}=$

$A \sqrt{\lambda-\frac{1}{\lambda}}$

$k=-4 A C+\lambda^{2}\left(4 A C-B^{2}\right)$
CaseII: $a_{0}=B \sqrt{\lambda-\frac{1}{\lambda}}, a_{1}=C \sqrt{\lambda-\frac{1}{\lambda}}$

$b_{1}=A \sqrt{\lambda-\frac{1}{\lambda}}$

$k=\left(1-\lambda^{2}\right)\left(-4 A C+\frac{C B^{2}}{A}-6 B^{2}\right)$

CaseIII: $a_{0}=\frac{B \sqrt{\lambda^{2}-1}}{2 \lambda}$,

$a_{1}=c \sqrt{\lambda^{2}-1}, b_{1}=0$

$k=\frac{\left(\lambda^{2}-1\right)}{2 \lambda}\left(-4 \lambda^{2} A C+B^{2}\right)$

The solition - like solutions to

\section{Zoomeron equation:}

1)When $\mathrm{A}=0, \mathrm{~B}=1, \mathrm{C}=-1$, from Table 1 ,

$F(\delta)=\frac{1}{2}+\frac{1}{2} \tanh \left(\frac{1}{2} \delta\right)$

By Case I, the exact Solution to equation (3-1) is given by:

$u_{1}=\frac{-1}{2} \sqrt{\lambda-\frac{1}{\lambda}}\left(1+\tanh \left(\frac{1}{2} x+\frac{1}{2} y-\frac{1}{2} \lambda t\right)\right)$

By Case II, the exact solutions to equation (3-1) are given by:

$u_{2}=\frac{1}{2} \sqrt{\lambda-\frac{1}{\lambda}}\left(1-\tanh \left(\frac{1}{2} x+\frac{1}{2} y-\frac{1}{2} \lambda t\right)\right)$

By Case III, the exact solutions to equation (3-1) are given by:

$u_{3}=\frac{\sqrt{\lambda^{2}-1}}{2}\left(\frac{1-\lambda}{\lambda}-\tanh \left(\frac{1}{2} x+\frac{1}{2} y-\frac{1}{2} \lambda t\right)\right)$

2) When $\mathrm{A}=0, \mathrm{~B}=-1, \mathrm{C}=1$, from Table $1, F(\delta)=$ $\frac{1}{2}-\frac{1}{2} \operatorname{coth}\left(\frac{1}{2} \delta\right)$

By Case I, the exact solutions to equation (3-1) is given by

$u_{4}=\frac{1}{2} \sqrt{\lambda-\frac{1}{\lambda}}\left(1-\operatorname{coth}\left(\frac{1}{2} x+\frac{1}{2} y-\frac{1}{2} \lambda t\right)\right)$

By Case II, the exact solutions to equation (3-1) is given by

$u_{5}=\frac{-1}{2} \sqrt{\lambda-\frac{1}{\lambda}}\left(1-\operatorname{coth}\left(\frac{1}{2} x+\frac{1}{2} y-\frac{1}{2} \lambda t\right)\right)$

By Case III, the exact solutions to equation (3-1) is given by

$\left.u_{6}=\frac{\sqrt{\lambda^{2}-1}}{2}\left(\frac{\lambda-1}{\lambda}-\operatorname{coth}\left(\frac{1}{2} x+\frac{1}{2} y-\frac{1}{2} \lambda t\right)\right)\right)$

3)When $\mathrm{A}=\frac{1}{2}, \mathrm{~B}=0, \mathrm{C}=\frac{-1}{2}$, from Table 1 ,

$F(\delta)=\operatorname{coth}(\delta) \pm \operatorname{Csch}(\delta)$

$F(\delta)=\tanh (\delta) \pm i \operatorname{sech}(\delta)$

By Case I, the exact solutions to equation (3-1) is given by

$u_{7}=\frac{-1}{2} \sqrt{\lambda-\frac{1}{\lambda}}(\operatorname{coth}(x+y-\lambda t) \pm \operatorname{Csch}(x+$

$y-\lambda t))$

$+\frac{1}{2} \sqrt{\lambda-\frac{1}{\lambda}}(\operatorname{coth}(x+y-\lambda t) \pm \operatorname{Csch}(x+y-$

$\lambda t))^{-1}$, 
$u_{8}=\frac{-1}{2} \sqrt{\lambda-\frac{1}{\lambda}}(\tanh (x+y-\lambda t) \pm i \operatorname{sech}(x+$ $y-\lambda t))$

$+\frac{1}{2} \sqrt{\lambda-\frac{1}{\lambda}}(\tanh (x+y-\lambda t) \pm i \operatorname{sech}(x+y-$ $\lambda t))^{-1}$.

By Case II, the exact solutions to equation (3-1) is given by

$u_{9}=\frac{-1}{2} \sqrt{\lambda^{2}-1}(\operatorname{Coth}(x+y-\lambda t) \pm \operatorname{Csch}(x+$ $y-\lambda t)$ ),

$u_{10}=\frac{-1}{2} \sqrt{\lambda^{2}-1}(\tanh (x+y-\lambda t) \pm i \operatorname{sech}(x+$ $y-\lambda t))$

4)When $\mathrm{A}=1, \mathrm{~B}=0, \mathrm{C}=-1$, from Table $1, \quad F(\delta)=$ $\tanh (\delta)$

$F(\delta)=\operatorname{coth}(\delta)$

By Case I, the exact solutions to equation (3-1) is given by

$u_{11}=-\sqrt{\lambda-\frac{1}{\lambda}}(\tanh (x+y-\lambda t)+$

$\sqrt{\lambda-\frac{1}{\lambda}}(\tanh (x+y-\lambda t))^{-1}$

$u_{12}=-\sqrt{\lambda-\frac{1}{\lambda}}(\operatorname{Coth}(x+y-\lambda t)+$

$\sqrt{\lambda-\frac{1}{\lambda}}(\operatorname{coth}(x+y-\lambda t))^{-1}$

By Case II, the exact solutions to equation (3-1) is given by

$u_{13}=-\sqrt{\lambda-\frac{1}{\lambda}}[\tanh (x+y-\lambda t)-$

$\left.(\tanh (x+y-\lambda t))^{-1}\right]$

$u_{14}=-\sqrt{\lambda-\frac{1}{\lambda}}[\operatorname{Coth}(x+y-\lambda t)-$

$\left.(\operatorname{Coth}(x+y-\lambda t))^{-1}\right]$

By Case III, the exact solutions to equation (3-1) is given by

$u_{15}=-\sqrt{\lambda^{2}-1}(\tanh (x+y-\lambda t)$

$u_{16}=-\sqrt{\lambda^{2}-1}(\operatorname{Coth}(x+y-\lambda t)$

The Trigonometric Function solutions to Zoomeron equation:

1)When $\mathrm{A}=\frac{1}{2}, \mathrm{~B}=0, \mathrm{C}=\frac{1}{2}$, from Table 1 ,

$F(\delta)=\sec (\delta)+\tanh (\delta)$

or $F(\delta)=\operatorname{Csc}(\delta)-\cot (\delta)$.

By Case I, the exact solutions to equation (3-1) is given by

$u_{17}=\frac{1}{2} \sqrt{\lambda-\frac{1}{\lambda}}[(\operatorname{Sec}(x+y-\lambda t)+\tan (x+y-$

$\lambda t))+\left(\operatorname{Sec}(x+y-\lambda t)+\tan (x+y-\lambda t)^{-1}\right]$,

$u_{18}=\frac{1}{2} \sqrt{\lambda-\frac{1}{\lambda}}[(\operatorname{Csc}(x+y-\lambda t)-\operatorname{Cot}(x+y-$

$\lambda t))+\left(\operatorname{Csc}(x+y-\lambda t)-\operatorname{Cot}(x+y-\lambda t)^{-1}\right.$.
By Case III, the exact solutions to equation (3-1) is given by

$u_{19}=\frac{1}{2} \sqrt{\lambda^{2}-1}(\operatorname{Sec}(x+y-\lambda t)+$

$\tan (x+y-\lambda t))$,

$u_{20}=\frac{1}{2} \sqrt{\lambda^{2}-1}(\operatorname{Csc}(x+y-\lambda t)+$

$\operatorname{Cot}(x+y-\lambda t))$.

2) When $\mathrm{A}=1, \mathrm{~B}=0, \mathrm{C}=1$, from Table 1 ,

$F(\delta)=\tan (\delta)(\cot (\delta))$

By Case I, the exact solutions to equation (3-1) is given by

$u_{21}=\sqrt{\lambda-\frac{1}{\lambda}} \tan (x+y-\lambda)(\operatorname{Cot}(x+y-\lambda))+$ $\sqrt{\lambda-\frac{1}{\lambda}}\left[\tan (x+y-\lambda)(\operatorname{Cot}(x+y-\lambda)]^{-1}\right.$

By Case III, the exact solutions to equation (3-1) is given by

$u_{22}=\sqrt{\lambda^{2}-1} \operatorname{Sec}(x+y-\lambda t)(\operatorname{Cot}(x+y-$ $\lambda)$ )

\section{The rational solution to zoomeron equation:}

1) When $\mathrm{A}=\mathrm{B}=0, C \neq 0$, from Table $1, F(\delta)=$ $\frac{1}{c \delta+\eta}(\eta$ is an arbitrary constant)

By Case I, an exact solutions to equation (3-1) can be written as:

$\mathrm{u}_{23}=\frac{-\mathrm{C} \sqrt{\lambda-\frac{1}{\lambda}}}{\mathrm{C}(\mathrm{x}-\lambda \mathrm{t})+\eta}$

By Case III, the exact solutions to equation (3-1) can be written as:

$u_{24}=\frac{-C \sqrt{\lambda^{2}-1}}{C(x-\lambda t)+\eta}$

2) When $A$ is an arbitrary constant, $B=C=0$, from Table $1, \mathrm{~F}(\delta)=\mathrm{A} \delta$,

By Case II, the exact solutions to equation (3-1) can be written as:

$u_{25}=\frac{\sqrt{\lambda-\frac{1}{\lambda}}}{(x-\lambda t)}$.

\section{The exponential Solution zoomeron} equation:

When $\mathrm{A}$ is an arbitrary constant, $\mathrm{B} \neq 0, \mathrm{C}=$ 0 , from Table $1, F(\delta)=\frac{\exp (B \delta)-A}{B}$.

By Case I, the exact solutions to equation (3-1) can be obtained that:

$u_{26}=A \sqrt{\lambda-\frac{1}{\lambda}}\left(\frac{\exp (B(x-\lambda t)-A)}{B}\right)^{-1}$.

By Case II, the exact solutions to equation (3-1) can be obtained that:

$u_{27}=B \sqrt{\lambda-\frac{1}{\lambda}}+A \sqrt{\lambda-\frac{1}{\lambda}}\left(\frac{\exp (B(x-\lambda t))-A}{B}\right)^{-1}$. 


\section{4- Conclusion}

The powerful modified F-expansion method was employed of the $(2+1)$ - dimensional zoomeron equation. This method is an efficient way to solve nonlinear PDES as it is used to solve differential equations which can be integrated or nonintegrated. By using this method, we have been able to calculate many new exact solutions for nonlinear partial differential equation include soliton-like solutions, trigonometric function solutions, rational solutions and exponential solutions.

\section{References}

[1]Ablowitz, m.J\& Clarkson, p.A. (1991), Nonlinear Evolution Equations and Inverse scattering, Cambridge university press.

[2]Akbarzade,M.\&Langari,J. (2011),Application of homotopy- perturbation method and variational iteration method to three dimensional diffusion problem, Int. Jour. Math.

[3]Bin zheng, J. (2012), (G'/G)-expansion method for solving Fractional partial differential equations in the Theory of Mathmatical physics commun, Theor. Phys.
[4]Caloger, F. \&Degasperis, A. (1982), Spectral transform and soitons I, North - Holland, New york.

[4]Caloger, F. \&Degasperis, A. (1982), Spectral transform and soitons I, North - Holland, New york.

[5]Gepreel, K.A,(2014), Explicit Jacobi Elliptic exact solutions for nonlinear partial fractional differential equations, Advanced Difference Equations.

[6]Ji , J. ,Wu, J. \& zhang, J. (2010),

Homogeneous balance method for an inhomogeneous kdv equation: Bäcklund transformation and Lax pair, Int. J. Nonlinear sci.

[7]Yan,C.T.(1996), A simple transformation for Nonlinear waved, Phys let A .

[8]Wang ,x. \&Yong. C. (2014), Darbonx Transformations and $\mathrm{N}$-soliton solutions of (2+1) - dimensional Nonlinear Equation, Commun. Theory . Phys .

[9]Wang, M.\& Li, X. (2005), Extended Fexpansion and periodic wave solutions for the generalized Zakharov equations, Phys. Let . A

\title{
الحلول المضبوطة لمعادلة تفاضلية جزئية لاخطية باستخدام طريقة F-expansion
}

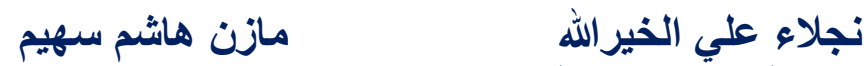 \\ جامعة سومر - كلية الادارة والاقتصاد - قسم الاحصاء \\ Mazin.suhhiem@yahoo.com nalkhairalla5@gmail.com
}

\author{
المستخلص: \\ في هذه الورقة،نستخدم طريقة F-expansion المعدلة لإيجاد حلول مضبوطة الإنديدة لمعادلة

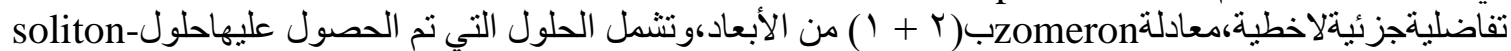

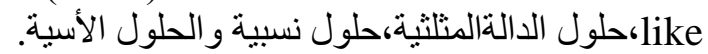

